\title{
El nuevo corte estratigráfico del yacimiento paleolítico de San Isidro (Madrid) de Emilio Rotondo sito en la Cátedra de Geología de la Escuela de Ingenieros de Caminos de Madrid
}

\author{
A stratigraphic section from the Palaeolithic site of San Isidro (Madrid) made by Emilio \\ Rotondo and located in the Civil Engineering Institute of Madrid
}

\author{
Eugenio Sanz Pérez ${ }^{\mathrm{a}}$ Ignacio Menéndez-Pidal de Navascués ${ }^{\mathrm{a}}$
}

\section{RESUMEN}

Se da cuenta de un nuevo tablero del corte estratigráfico del yacimiento paleolítico de San Isidro realizado por Emilio Rotondo. Conserva la única descripción detallada de su estratigrafía, lo que contribuye a mejorar la información de este histórico yacimiento. Se identifican 34 niveles por sus características granulométricas, color, espesor y estructura. Dos de ellos tenían material arqueológico: la capa 26 de arenas y arcillas con industria lítica y restos de mamíferos fósiles (también las capas inferiores 27-28-29-30) y la capa 34 de gravas con industria lítica.

\begin{abstract}
We present a new section by Emilio Rotondo from the Palaeolithic site of San Isidro that preserves the only detailed description of its stratigraphy and thus improves our knowledge about this historical site. 34 levels of different particle size, color, thickness and structure are identified. There are two differentiated archaeological levels: an upper one (layer 26) formed by sands, clays and containing a lithic industry and remains of fossil mammals (these are also found in layers 27 through 30) and a lower one (layer 34) formed by gravels with lithic industry.
\end{abstract}

Palabras clave: Historiografía; Siglo XIX; Material docente; Paleolítico inferior; Achelense; Yacimiento de San Isidro; Terraza río Manzanares; Madrid; España.

Key words: Historiography; 19th Century; Educational Material; Lower Paleolithic; Acheulean; San Isidro; Manzanares River Terrace; Madrid; Spain.

\section{INTRODUCCION Y OBJETIVOS}

La Escuela de Ingenieros de Caminos de Madrid ha tenido una importancia destacable en el desarrollo científico-técnico español desde su fundación a finales del siglo XVIII hasta el tiempo presente, con figuras de la talla de Leonardo Torres Quevedo o José Echegaray, por ejemplo (Sáenz Ridruejo 1998, 2006). Además los ingenieros de caminos, por el carácter de sus trabajos, realizaron aportaciones sustantivas al avance de los estudios arqueológicos y prehistóricos en la España del siglo XIX. Es el caso de Eduardo Saavedra, descubridor de Numancia (Mañas Martínez 1983) o de José Subercase, que formó parte de la primera comisión que levantó en 1849 la

a Cátedra de Geología, Dpto. de Ingeniería y Morfología del Terreno, Escuela Técnica Superior de Ingenieros de Caminos, Canales y Puertos. Universidad Politécnica de Madrid. Ciudad Universitaria. C/ Profesor Aranguren s/n. 28040 Madrid.

Correos e.: esanz@caminos.upm.es; ignacio.menendezpidal@upm.es http://orcid.org/0000-0002-7053-1101

Recibido 14-VI-2016; aceptado 21-IX-2016. 
carta geológica de Madrid junto con ingenieros de minas y naturalistas (Rábano 2015).

El laboratorio de la Cátedra de Geología de la Escuela de Ingenieros de Caminos de Madrid conserva algunas colecciones del Paleolítico recopiladas desde su fundación en 1802 hasta la actualidad por algunos de sus profesores. En su formación influyó que la Geología, la Paleontología y la Arqueología prehistórica fueran materias impartidas por ellos, lo que también ocurría con los profesores de la Escuela de Minas (Puche 1993). Entre las investigaciones arqueológicas importantes citamos, a modo de ejemplo, los primeros estudios del yacimiento de la Edad del Bronce de Totana (Murcia), descubierto por el catedrático de Geología de la Escuela de Ingenieros de Caminos, Rogelio de Inchaurrandieta.

A principios del siglo pasado una expresión del interés por la geología del Cuaternario son las visitas de los alumnos de dicha Escuela a los yacimientos prehistóricos del Campo de San Isidro durante todos los cursos. Eran parte de las lecciones prácticas de la asignatura de Estratigrafía y Física Terrestre a cargo de los catedráticos de Geología, Narciso Puig de la Bellacasa y Francisco Hernández-Pacheco que, como se sabe, fue fundador y director de la Comisión de Investigaciones Paleontológicas y Prehistóricas de la Junta para la Ampliación de Estudios e Investigaciones Científicas. También se enseñaba a los estudiantes las colecciones de objetos prehistóricos y del Cuaternario del Museo Antropológico de Madrid, donde eran atendidos por el profesor Manuel Antón (Anuario de Curso, años 1907 a 1936). No es extraño por tanto que, entre las colecciones existentes en la Escuela de Ingenieros de Caminos, se conservase uno de los tableros del corte estratigráfico de San Isidro realizado por Emilio Rotondo y Nicolau, que sirvió sin duda como recurso y material docente. El estudio de las formaciones superficiales cuaternarias está doblemente justificado en el ámbito profesional de la ingeniería civil. Son las formaciones geológicas que más interaccionan con las obras públicas lineales y, además, areneros como los de la terraza de ese yacimiento son fuente de materiales para la construcción.

La colección de objetos prehistóricos formada por Emilio Rotondo que había estado largo tiempo depositada en el Museo Arqueológico Nacional pasó a la Dirección de Investigaciones Prehistóri- cas del Ayuntamiento de Madrid. Allí José Pérez de Barradas la estudió en 1925 (Pérez de Barradas 1929), algo después de su trabajo en el propio yacimiento (Wernert y Pérez de Barradas 1925; Salas Vázquez 2008).

Emilio Rotondo realizó varios cortes similares (Cacho y Martos 2002). Se conservan al menos tres en España. El objetivo de este trabajo es dar a conocer un cuarto, el tablero de la Escuela Especial de Ingenieros de Caminos, y explicar en detalle la estratigrafía representada, ya que es el único que sepamos, que guarda la información escrita adicional. Al poder interpretar mejor los estratos se realza la utilidad de los cortes ya identificados del autor que son similares aunque no iguales.

El renombrado e histórico yacimiento paleolítico de San Isidro se halla situado en la terraza del río Manzanares que le da nombre. La base de esta terraza se sitúa hacia las cotas $+35-40 \mathrm{~m}$. Tiene el mérito de ser el primer yacimiento paleolítico descubierto en España, pero su excavación poco sistemática ha limitado su aportación al conocimiento del Paleolítico. A mediados del siglo XIX el yacimiento estaba muy mermado (Pérez de Barradas 1941) y actualmente se le considera agotado. Gamazo et al. (2001) y Gómez et al. (2005) aportan nuevos datos sobre el Paleolítico de la terraza. El material recogido y revisado con posterioridad tiene pocas referencias estratigráficas (Aguirre 2002; Gamazo 2002) pero ha sido posible establecer dos tipologías en la industria lítica: una con bifaces amigdaloides situada en los niveles inferiores y otra con bifaces cordiformes lanceolados u ovales que parece corresponder a niveles superiores (Santonja 1977; Santonja y Vega 2002). La industria lítica pertenece al Achelense (Pleistoceno Medio) y es correlacionable con el yacimiento Aridos-1. Por otro lado, en los niveles inferiores y medios de San Isidro se recogieron Bovidae ind., Cervus sp., Equus caballus y Elephas (Palaeoloxodon) antiquus platyrhinchus (Hernández Pacheco 1927; Aguirre 1969; Sesé y Soto 2002a, b).

\section{METODOLOGÍA}

El Laboratorio de Geología de la Escuela Técnica Superior de Ingenieros de Caminos, Canales y Puertos ha emprendido la investigación de las 
colecciones de rocas, fósiles, minerales y material arqueológico, recopilados desde la fundación de la Escuela en 1802 hasta la actualidad. Ello ha incluido su inventario y catalogación, así como su documentación e interpretación histórica. Los útiles paleolíticos parece que son posteriores a
1863, aunque no lo podemos asegurar. Entre los materiales relacionados con el Paleolítico destaca el mencionado corte estratigráfico del yacimiento de San Isidro, realizado por Emilio Rotondo Nicolau (Fig. 1), una pieza de alto valor historiográfico (inventariado como Mapas y Cortes, $\mathrm{n}^{\mathrm{o}} 1$ Rotondo).
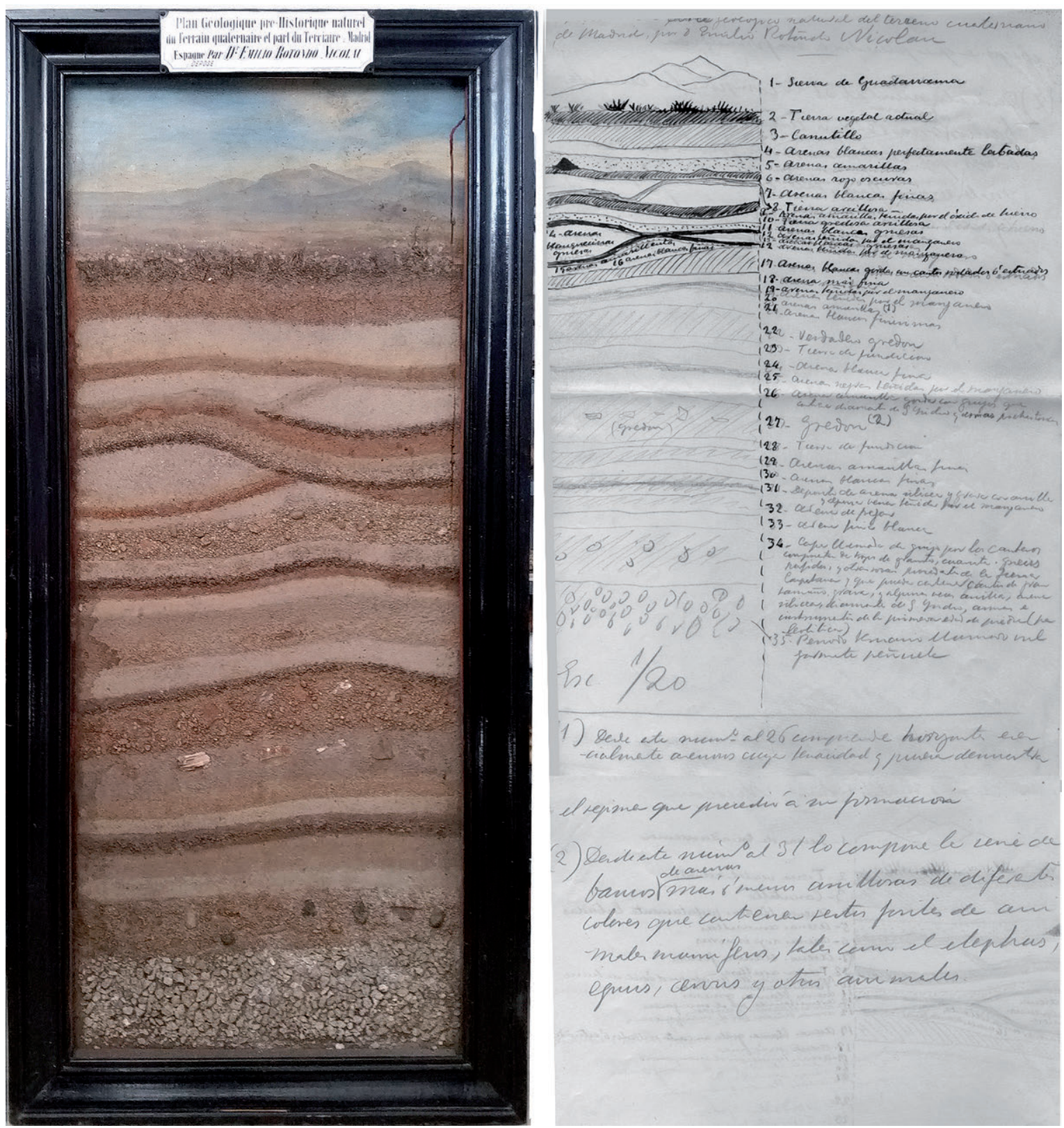

Fig. 1. Tablero del corte estratigráfico del yacimiento paleolítico de San Isidro realizado por Emilio Rotondo Nicolau, adquirido antes de 1927 por la Escuela de Ingenieros de Caminos, Canales y Puertos de Madrid, donde se conserva inventariado como Mapas y Cortes, $\mathrm{n}^{\circ} 1$ Rotondo. Mide 1,75 m de alto por 0,67 m de ancho. Fotografía E. Sanz e I. Menendez-Pidal

Trab. Prehist., 73, N. ${ }^{\circ}$ 2, julio-diciembre 2016, pp. 304-314, ISSN: 0082-5638 doi: $10.3989 /$ tp.2016.12175 
Durante la catalogación aparecieron documentos desordenados de carácter docente asociados a algunos de esos materiales. Un sobre del curso 1927-1928 contenía unas notas manuscritas de Rotondo con la explicación detallada de la columna estratigráfica del corte, así como unos dibujos interpretativos del tablero (Fig. 2). Todo ello parece indicar que fue adquirido en ese curso. Como dato curioso, en el sobre estaba también la lista de asistencias y notas de los alumnos matriculados en la asignatura de Geología del segundo curso en la Escuela Especial de Ingenieros de Caminos de Madrid, donde aparece Emeterio Cuadrado Diez, ingeniero de caminos y renombrado arqueólogo.

Durante la investigación se ha identificado la descripción, escrita por Emilio Rotondo, con la columna estratigráfica del tablero que no tenía cada capa numerada. El texto distingue 34 niveles que han podido ser identificados en el "cuadro", aunque el color de los sedimentos en algunos haya perdido intensidad o estén sucios por el polvo. La identificación no es tan simple en los estratos superiores que tienen estratificación cruzada pero los apuntes manuscritos aclaratorios de la época y que se reproducen en la figura 1 han servido para lograrla. La columna estratigráfica resultante se ha relacionado y comparado posteriormente con el resto de la documentación más importante publicada de carácter estratigráfico e histórico. Hemos recurrido a la primera descripción de Casiano del Prado $(1864,1866)$, a la de Vilanova (1872b) en base a estos mismos tableros, y a la de la Comisión del Mapa Geológico de España (Graells 1897).
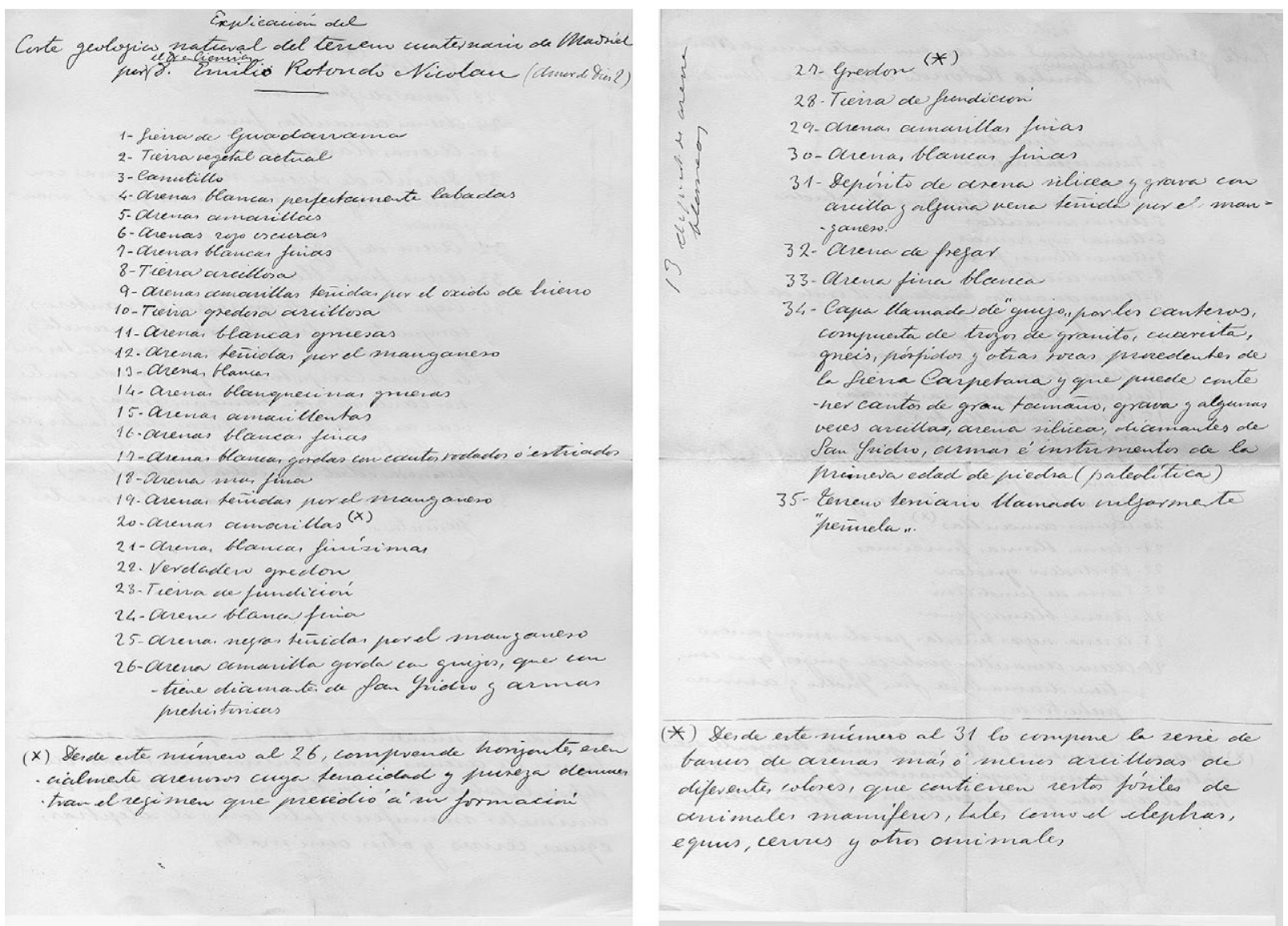

Fig. 2. Manuscritos y esquemas originales de la explicación de los niveles estratigráficos del yacimiento paleolítico de San Isidro, representados en el tablero de Emilio Rotondo que se conserva en la Escuela de Ingenieros de Caminos, Canales y Puertos de Madrid (curso 1927-1928). Fotografía E. Sanz e I. Menendez-Pidal 


\section{RESULTADOS}

\subsection{Descripción del tablero}

El tablero mide $1,75 \mathrm{~m}$ de alto por $0,67 \mathrm{~m}$ de ancho y conserva el marco negro de madera original. Le protegía un cristal actualmente inexistente. En la parte de arriba hay un letrero en francés que traducimos: "Plano geológico prehistórico natural del Terreno cuaternario y Terciario de Madrid, España, por D. Emilio Rotondo Nicolau". El empleo del francés no es de extrañar ya que en la segunda mitad del siglo XIX era la lengua científica internacional. Su estado de conservación no es malo, tal como se puede apreciar en la figura 1, aunque está sucio de polvo y presenta una raja lateral en un lado. Incluso creemos que es el mejor conservado de los existentes, ya que no se han hecho modificaciones, ni añadidos y presenta intactas las litologías de las diferentes capas, y un número apreciable de industria lítica y restos óseos.
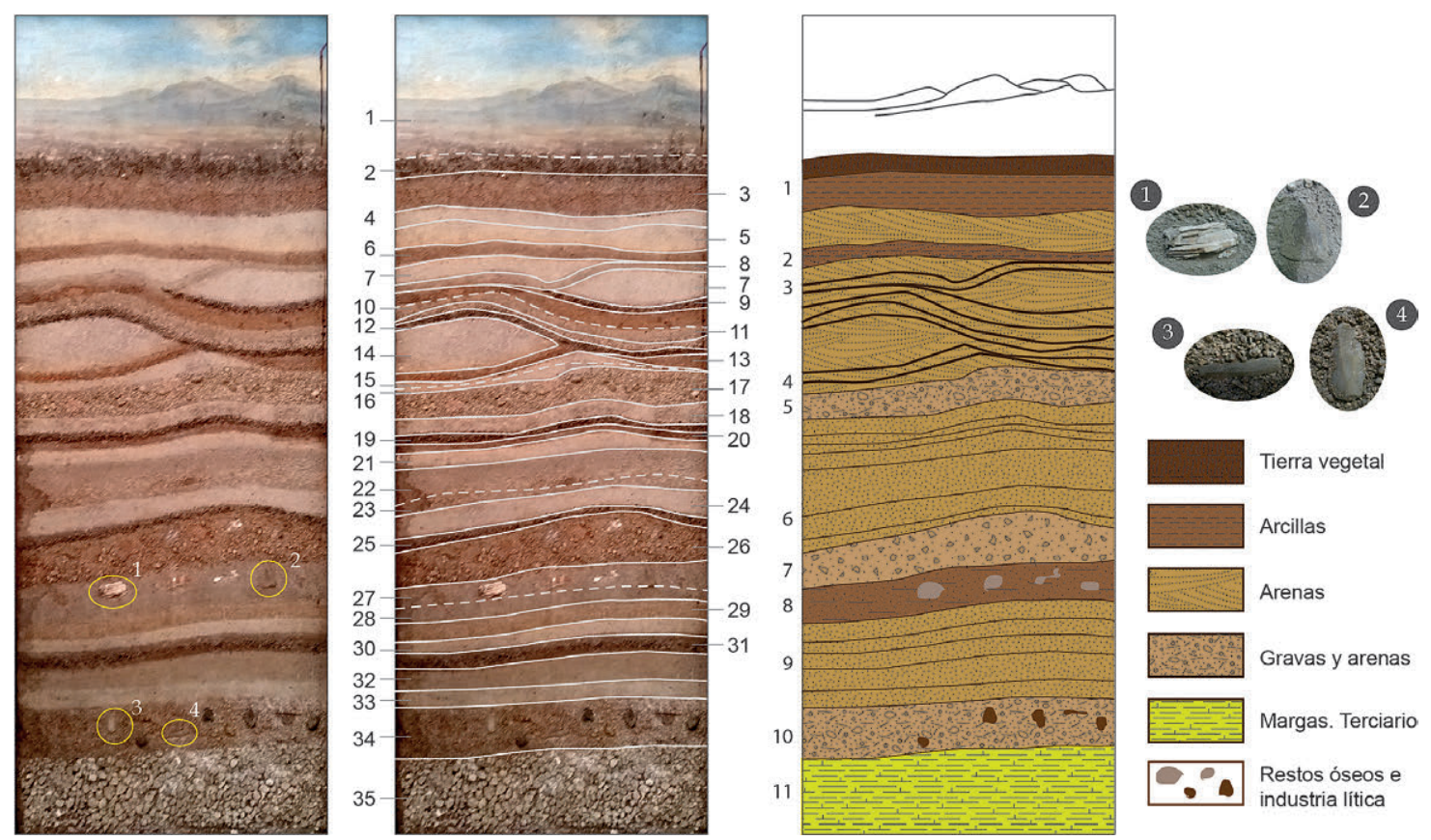

Fig. 3. Interpretación estratigráfica del tablero de Emilio Rotondo conservado en la Escuela de Ingenieros de Caminos, Canales y Puertos de Madrid: izquierda fotografía del tablero original; centro identificación de los 35 niveles estratigráficos explicados por Emilio Rotondo; derecha identificación de las 12 capas descritas por Vilanova (1872). Detalles de la industria lítica paleolítica y de las piezas paleontológicas adheridas: 1. Molar de Equus muy fragmentado; 2 . a 4. Piezas de sílex. Fotografía E. Sanz e I. Menendez-Pidal.

Trab. Prehist., 73, N. ${ }^{\circ} 2$, julio-diciembre 2016, pp. 304-314, ISSN: 0082-5638

doi: $10.3989 /$ tp.2016.12175 


\subsection{Descripción de los niveles estratigráficos}

Se caracteriza a continuación la sucesión de niveles de arriba abajo y, teniendo en cuenta la escala 1/20 del plano, se les asigna entre paréntesis los espesores reales en metros: 1 . Sierra de Guadarrama. 2. Tierra vegetal actual $(0,7 \mathrm{~m}) .3$. Canutillo $(1,5 \mathrm{~m})$. 4. Arenas blancas perfectamente lavadas $(0,4 \mathrm{~m}) .5$. Arenas amarillas $(0,7 \mathrm{~m}) .6$. Arenas rojas oscuras $(0,5 \mathrm{~m})$. 7. Arenas blancas finas $(1,10 \mathrm{~m}) .8$. Tierra arcillosa $(0,3 \mathrm{~m}) .9$. Arenas amarillas teñidas por el óxido de hierro $(0,7 \mathrm{~m})$. 10. Tierra gredosa amarilla $(0,4 \mathrm{~m})$. 11. Arenas blancas gruesas $(0,2 \mathrm{~m}) .12$. Arenas teñidas por el manganeso $(0,2 \mathrm{~m})$. 13. Arenas blancas (entre 0 y $0,6 \mathrm{~m}$ ya que es un lentejón). 14. Arenas blanquecinas gruesas (entre 0 y $1,8 \mathrm{~m}$ ya que es un lentejón). 15. Arenas amarillentas (0,2 m). 16. Arenas blancas finas (entre 0 y $0,6 \mathrm{~m})$. 17. Arenas blancas gordas con cantos rodados estriados (1 m). 18. Arena más fina $(0,5 \mathrm{~m})$. 19. Arenas teñidas por el manganeso $(0,2 \mathrm{~m})$. 20. Arenas amarillas ${ }^{1}$ $(0,2 \mathrm{~m})$. 21. Arenas blancas finísimas $(0,5 \mathrm{~m})$. 22. Verdadero gredón $(1 \mathrm{~m})$. 23. Tierra de fundición $(0,6 \mathrm{~m}) .24$. Arena blanca, fina $(0,7 \mathrm{~m}) .25$. Arenas negras teñidas por el manganeso $(0,1 \mathrm{~m})$. 26. Arena amarilla gorda con guijos, que contiene diamantes de San Isidro y armas prehistóricas (1,6 $\mathrm{m})$. 27. Gredón ${ }^{2}(1,5 \mathrm{~m})$. 28. Tierra de fundición $(0,4 \mathrm{~m})$. 29. Arenas amarillas finas $(0,6 \mathrm{~m}) .30$. Arenas blancas finas $(0,5 \mathrm{~m})$. 31. Depósito de arena silícea y grava con arcilla y alguna vena teñida por el manganeso $(0,6 \mathrm{~m})$. 32. Arena de fregar $(0,9 \mathrm{~m}) .33$. Arena fina blanca $(0,6 \mathrm{~m}) .34$. Capa llamada de "Guijo" por los canteros compuesta de trozos de granito, cuarcita, gneis, pórfidos y otras rocas procedentes de la Sierra Carpetana y que puede contener cantos de gran tamaño, grava y algunas veces arcilla, arena silícea, diamantes de San Isidro, armas e instrumentos de la primera edad de piedra (paleolítica) $(1,8 \mathrm{~m}) .35$. Terreno terciario llamado vulgarmente "peñuela" (3,2 m espesor visto).

\footnotetext{
${ }^{1}$ Desde este número al 26 comprende horizontes esencialmente arenosos cuya tenacidad, y pureza demuestran el régimen que precedió á su formación.

2 Desde este número al 31 lo compone la serie de bancos de arenas más o menos arcillosas de diferentes colores, que contienen restos fósiles de animales mamíferos, tales como el Elephas, Equus, Cervus y otros animales.
}

\section{DISCUSIÓN}

\subsection{Sobre los tableros del corte estratigráfico del yacimiento de San Isidro realizados por Emilio Rotondo}

Emilio Rotondo (1849-1916) fue un personaje singular y hombre de su época con notables aficiones arqueológicas (Ariño 2007; Sánchez Miñana y Villanueva 2014). Se le conocía como geólogo y arqueólogo, en cuyas temáticas se inició gracias a Juan de Vilanova y Piera. Sin embargo la sistematización de la Geología y la Arqueología y la depuración de los métodos de excavación de los yacimientos provocaron críticas y recelos científicos hacia sus colecciones (Wernert y Pérez de Barradas 1925; López Rodríguez 2015).

Ya indicamos que Emilio Rotondo realizó varios cortes similares, aunque ninguno es igual a otro. Esta obra gráfica de carácter arqueológico se mostró en la Exposición del Congreso Internacional de Prehistoria y Antropología de Copenhague (1869), en la Exposición Internacional de Londres (1871), en la Exposición Universal de París (1878), en la Exposición Nacional de Madrid (1873) y en la XIII Exposición Internacional de Burdeos (1895) (Cacho y Martos 2002). Emilio Rotondo no aparece inscrito en el Congreso de Copenhague, aunque sí lo están Rogelio de Inchaurrandieta, Juan Vilanova y Francisco María Tubino. No se sabe quién de ellos llevaría a Dinamarca el material de Rotondo, pero es significativo que en dicho congreso estuviera el catedrático de Geología de la Escuela Especial de Ingenieros de Caminos, Rogelio de Inchaurrandieta. Como se ve, Emilio Rotondo fue un difusor temprano a escala internacional del yacimiento de San Isidro. $\mathrm{Su}$ obra fue reconocida en el ámbito científico dentro y fuera de España y fue objeto de varias menciones honoríficas en 1871 en la Exposición Internacional de Londres y en la Exposición Nacional Española. El corte fue además reproducido en publicaciones de Vilanova (1872a, b).

Estas representaciones gráficas de cortes estratigráficos fueron adquiridas por diversas instituciones, como las de la Comisión del Mapa Geológico de España para la exposición de Viena (1873), que no llegó finalmente a exhibirse, ya que sufrió deterioro, o por las principales universidades de España y Ultramar, como la de la 
Habana (1876), la Universidad Central de Madrid y los institutos madrileños de enseñanza secundaria de San Isidro y de Noviciado (Cacho y Martos 2002). De este corte estratigráfico se conocían dos ejemplares en Madrid, uno en el Museo de San Isidro y otro en el Museo Arqueológico Nacional, fechado en 1876 (Cacho y Martos, 2002), que guarda un gran parecido con este de la Escuela de Caminos de la Universidad Politécnica. También fueron adquiridos en el extranjero por instituciones como el Museo Británico y personalidades como el emperador de Brasil. Los cortes del Museo de San Isidro y del Museo Arqueológico Nacional tal vez provengan de las colecciones del Museo Protohistórico Ibérico (1897), fundado por Emilio Rotondo y situado en las Escuelas Aguirre también en Madrid. Tiempo después el Estado adquirió parte de la colección y la depositó en el Museo Antropológico Nacional, cediendo el resto al Ayuntamiento de Madrid, quien lo instaló en la Casa de Panadería, acabando después en el Museo de San Isidro

\subsection{Estratigrafía del tablero de Rotondo en el contexto histórico de la época}

Este corte estratigráfico ayuda a conocer los inicios de la Prehistoria en España de la que Emilio Rotondo debe considerarse como uno de los pioneros. Realizaría el primer corte solo cinco años después de la publicación de Casiano del Prado (1864), reflejando claramente la posición de la industria lítica en niveles situados por debajo de otros con restos de animales extintos. Con ello sigue las ideas modernas, formuladas en 1859, en la Sociedad Geológica de Londres y asumidas por la comunidad científica internacional. Además, utiliza los criterios paleontológicos de división del Cuaternario establecidos por Lartet en 1862.

La representación de las diferentes capas de terreno con los fósiles asociados a ellas es infrecuente en las publicaciones científicas de la época, pero no es del todo original de Rotondo. Buffon (1858), por ejemplo, incluye en su obra dos figuras de cortes geológicos de este estilo. Rotondo podría haber combinado esta inspiración en las obras geológicas de entonces con un rasgo original e inédito como parece ser el dispositivo visual consistente en un tablero con una columna estratigráfica hecha a base de elementos naturales de arenas y gravas del procedentes del yacimiento, incluyendo la industria lítica. Quizá podríamos relacionar esta peculiar representación con una época en que estaba emergiendo el poderoso lenguaje visual geológico (Rudwick 1976).

La potencia total de la estratigrafía que nos muestra este corte es de unos $20,7 \mathrm{~m}$. Las facies, mayoritariamente de arenas, se reparten en 34 capas de arenas, gravas, arcillas, limos, que se apoyan discordantemente sobre margas terciarias (Fig. 3). El espesor más corriente de las capas oscila entre $0,4-0,7 \mathrm{~m}$. El que sea el habitual en los sedimentos de esta terraza, según los registros de sondeos, da verosimilitud a esta representación histórica. Son facies de canal en el que las arenas se agrupan mayoritariamente en la parte superior y aparecen bancos de gravas de mayor energía y delgados horizontes de limos y arcillas. En la sedimentación del canal hay estratificaciones cruzadas, ondulaciones, acuñaciones por cambios laterales de facies, etc, fiel reflejo de un medio fluvial. Lo mismo ocurre en la representación estratigráfica realizada por la Comisión del Mapa Geológico de España de la figura 4 (Graells 1897: lám. XIX; véase nuestra tab. 1). A veces los sedimentos están teñidos de óxidos de hierro o de manganeso que indican que estos areneros constituyeron acuíferos, quedando reflejados los antiguos niveles del freático al quedar como terrazas colgadas. Esto también es bastante normal.

Siguiendo la explicación de los niveles estratigráficos de un tablero similar a este, citado en el Compendio de Geología de Vilanova (1872a), no es difícil identificar y correlacionar los diferentes horizontes con el de la Escuela de Caminos (Fig. 3). En los niveles definidos por Vilanova se han insertado las 34 capas de este tablero (Fig. 3, Tab. 1), añadiendo subdivisiones de detalle a la estratigrafía del yacimiento. También es posible correlacionar de manera aproximada los horizontes definidos por la Comisión del Mapa Geológico de España (Fig. 4), (según Graells 1897) con los de Vilanova, tal como se presenta en la tabla 1.

Por otra parte, las descripciones de Casiano del Prado (1864), identificando unos $12 \mathrm{~m}$ de espesor total, distinguiendo $7,8 \mathrm{~m}$ superiores de arenas, $0,5-1 \mathrm{~m}$ de greda intermedia y $3 \mathrm{~m}$ de guijo inferior suponen una síntesis de la estratigrafía del yacimiento, pero no contradicen sustancialmente

Trab. Prehist., 73, N. ${ }^{\circ}$ 2, julio-diciembre 2016, pp. 304-314, ISSN: 0082-5638

doi: $10.3989 /$ tp.2016.12175 


\begin{tabular}{|c|c|c|}
\hline COLUMNA A & $\begin{array}{c}\text { COLUMNA B } \\
(\text { Nota } 1)\end{array}$ & $\begin{array}{c}\text { COLUMNA C } \\
(\text { Nota } 2)\end{array}$ \\
\hline 2,3 & 1 & 1,2 \\
\hline $4,5,6,7$ & 2 & 3 \\
\hline $8,9,10,11,12,13,14,15,16$ & 3,4 & 4,5 \\
\hline 17 & 5 & 4,5 \\
\hline $18,19,20,21,22,23,24,25$ & 6 & 6,8 \\
\hline 26 & 7 & 7,8 \\
\hline 34 & 8 & 9,10 \\
\hline 35 & 9 & 11 \\
\hline & 10 & Ter. \\
\hline
\end{tabular}

Tab. 1. Intento de identificación y correlación entre las diferentes capas del tablero de Rotondo de la Escuela Especial de Ingenieros de Caminos (columna A) con las columnas estratigráficas del yacimiento paleolítico de San Isidro según Vilanova (1872, Columna B, Nota 1) y la Comisión del Mapa Geológico de España (Graells 1897, Columna C, Nota 2).

Nota 1. Descripción de Vilanova (1872).

1. Tierra vegetal y greda (arcilla arenosa de color rojizo) con un espesor conjunto de 2,5 a $3 \mathrm{~m}$. Gredón (nombre que daban los alfareros de San Isidro a las arcillas compactas azuladas) intercalado entre dos capas de arena.

3 y 4 . Horizontes delgados de arena silícea-feldespática y algo micáceas puras y blancas, o bien algo arcillosas de color rojizo dispuesto todo en estratificación cruzada propio del medio de sedimentación fluvial. Todo el conjunto tiene $3 \mathrm{~m}$ de espesor. Aparecen algunos niveles de arena horizontales y paralelos de color negro impregnados de manganeso que indican paleoniveles del freático. El conjunto tiene $3 \mathrm{~m}$ de espesor.

5. Capa de gravas de cantos rodados de tamaño pequeño (guijo) con matriz de arena arcillosa y silícea algo rojizas. Espesor de unos

$2 \mathrm{~m}$. este nivel no tiene restos orgánicos ni industria lítica.

6. Horizonte esencialmente de arena de 2 a $2,5 \mathrm{~m}$ de potencia.

7. Capa de gravas de cantos rodados de tamaño algo menor que la capa de guijo de arriba y sostenidos en arenas arcillosas. Espesor de 0 ' $5 \mathrm{~m}$. Aparece algún instrumento tosco de pedernal.

8. Banco de arenas arcillosas muy finas de color rojizo de entre 1 a $1,3 \mathrm{~m}$ de espesor. Constituye un nivel fértil de huesos y dientes de caballo, ciervo y otros mamíferos.

9. Depósito de $1,5 \mathrm{~m}$ de arena silícea y grava con arcilla, sin restos fósiles ni hachas. Aparece algún horizonte teñido de negro de Mn. 10. Capa de guijo (así lo llamaban los canteros de la zona) de $2 \mathrm{~m}$ que se haya formada por guijarros de granitos, pórfidos, cuarcita, gneis y otras rocas procedentes de la Sierra Carpetana, cuyo tamaño llega a superar los $30 \mathrm{~cm}$ de diámetro. Estos cantos se hayan mezclados con gravas, arena silícea y arcillas.

El nivel constituye el principal yacimiento de hachas de pedernal que tanta importancia han dado a la localidad, y de los cantos de cuarzo llamados por su trasparencia y pureza "diamantes de San Isidro".

11. Cayuela (marga blanca), que constituye el basamento terciario sobre el que se apoya en superficie de erosión el resto de los materiales cuaternarios.

Nota 2. Descripción de la Comisión del Mapa Geológico de España (Graells 1897).

1. Tierra vegetal: espesor, unos $40 \mathrm{~cm}$.

2. Arcilla arenosa parduzca de $2,5 \mathrm{~m}$ de espesor

3. Arena gruesa con venas de greda azul, de $75 \mathrm{~cm}$ de espesor

4. Arenas amarillento-rojizas feldespáticas y cuarzosas con alguna mica con frecuentes cantos rodados. Vetas de arcilla y manchas

de óxido de hierro y manganeso. Potencia de entre 5 y $6 \mathrm{~m}$

5. Arenas arcillosas gruesas con cordones de guijarros poco rodados. De 1,5 $\mathrm{m}$ de espesor.

6. Arenas gruesas rojizas compactas de $3,25 \mathrm{~m}$ de espesor.

7. Arcilla plástica azulada (gredón de los tejeros), de $85 \mathrm{~cm}$

8. Banco de 1,60 metros de espesor formado exclusivamente por arenas blancas muy finas. En este horizonte y el inmediato superior de gredón se han encontrado en diversas épocas restos de paquidermos fósiles.

9. Arenas y arcillas ferruginosas, con espesor de 90 centímetros.

10. Zona de 1,50 $\mathrm{m}$ de espesor constituido por gravas y arenas medianas, donde se señala los horizontes de los distintos acarreos por el predominio de las piedras o de las arenas.

11. Horizonte de 2 metros de guijo o cantos rodados, de granito, pórfido cuarcífero, cuarzo y feldespatos, envuelto por arenas gruesas... Entre los materiales se suelen encontrar cuarzo hialino (diamantes de San Isidro) y hachas de piedra.

12. Debajo de todo lo anteriormente citado se encuentran las peñuelas terciarias, y en el contacto de las dos formaciones abundan las aguas, como se comprende fácilmente dada la permeabilidad de la zona del guijo y la impermeabilidad de la marga miocena.

la presentada en este tablero, salvo en el espesor. En realidad, las diferencias estratigráficas entre los distintos autores no son tan importantes. Pueden deberse tanto a la lógica variabilidad estratigráfica de los medios fluviales que, aun estando en puntos relativamente próximos, tienen numerosos acuñamientos y capas lenticulares, como a las distintas situaciones de explotación del arenero cuando eran levantadas las columnas, diferentes referencias en las mediciones, etc. 


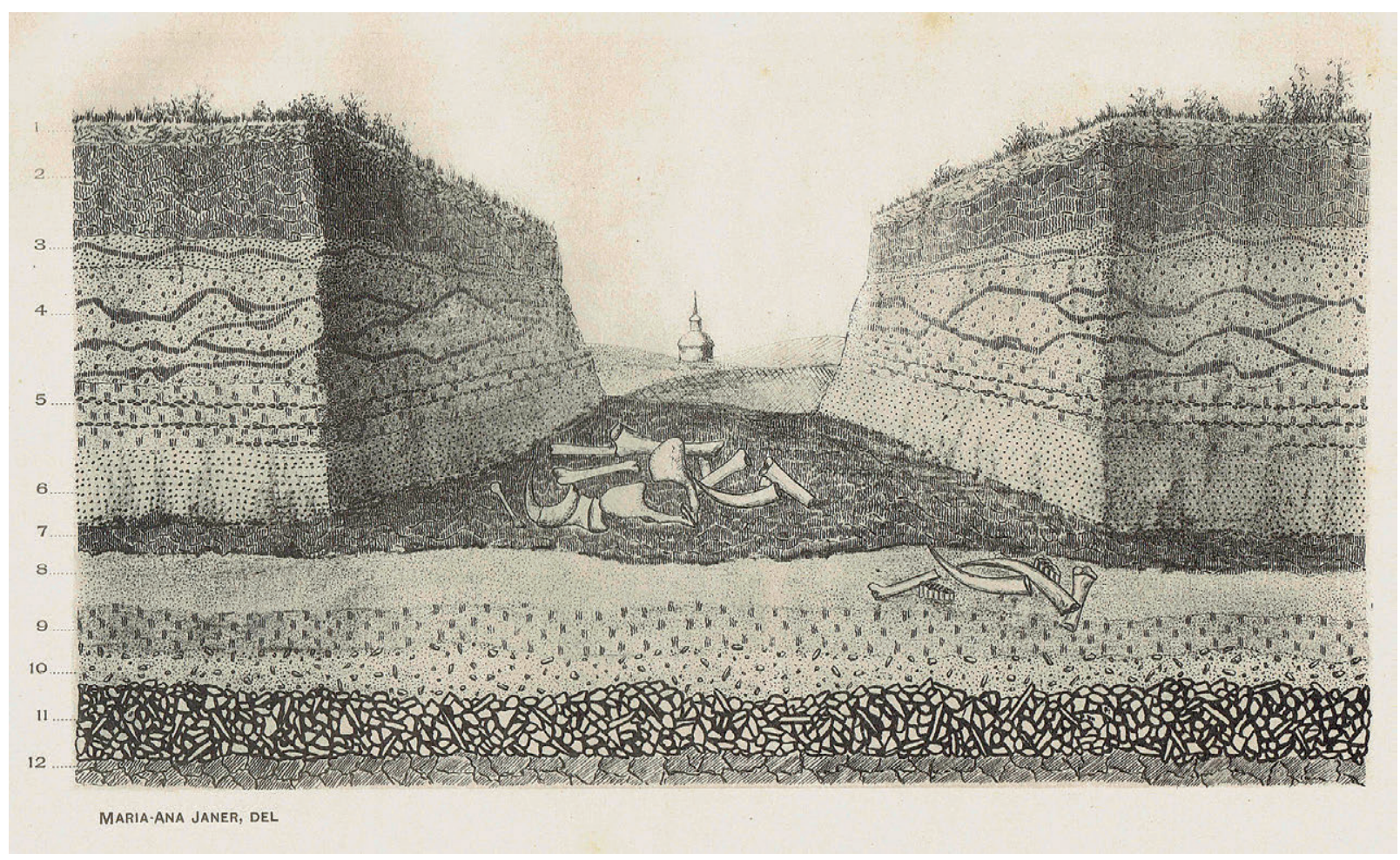

Fig. 4. Identificación de las 12 capas descritas por la Comisión del Mapa Geológico de España del yacimiento de San Isidro (según Graells 1897: Lámina XIX)

\subsection{Los niveles arqueológicos}

Según las notas que acompañan a este corte estratigráfico, parece que en él se distinguen dos niveles estratigráficos:

Nivel superior, constituido por un conjunto de 4,6 $\mathrm{m}$ de espesor formado por arenas y arcillas. Incluye la capa 26 con industria lítica, junto con las capas 27-28-29-30 con restos de mamíferos fósiles. Sin embargo, en el tablero solo se representan juntos los fósiles y la industria lítica en el nivel 27. Quizá haya que pensar en limitaciones de escala para no "empastar" la representación gráfica. En todo caso, parece que al autor no le repugnaba agruparlas y por eso aquí se unen.

Nivel inferior, construido por $1,8 \mathrm{~m}$ de gravas (capa 34) con industria lítica.

\subsection{Identificación y comparación de las unidades estratigráficas del tablero de}

\section{la Universidad Politécnica con las de los tableros del Museo Arqueológico Nacional y del Museo de San Isidro de Madrid}

El parecido del corte del Museo Arqueológico Nacional con el de la Universidad Politécnica es muy grande. Hay coincidencia salvo en cuatro niveles que seguramente existieron en el original, pero se perdieron por alguna diferencia en la restauración. El nivel 2 se agrupa con el 3 y los niveles 21, 22, 23 y 24 se reúnen en uno solo. Si se hubiera conocido la leyenda que se presenta en este trabajo, la restauración hubiera podido hacerse mejor. Sin embargo, gracias a ella, los colores de las capas son más reconocibles, en la descripción de la leyenda, que en el tablero de la Escuela de Ingenieros de Caminos. Por lo demás, los grosores de las capas son muy parecidos y la geometría de los estratos es también casi igual, aunque con pequeñas y lógicas variaciones. En el del Museo Arqueológico no se conservan restos óseos, como en este de la Escuela de Caminos.

Trab. Prehist., 73, N. ${ }^{\circ}$ 2, julio-diciembre 2016, pp. 304-314, ISSN: 0082-5638

doi: $10.3989 /$ tp.2016.12175 
El corte expuesto al público en el Museo de San Isidro sería seguramente bastante semejante también, pero ahora su estado original está bastante modificado al haberse simplificado la estratigrafía en 25 niveles y haberse utilizado en la restauración materiales a veces muy distintos de los originales del yacimiento.

\section{RECAPITULACIÓN}

Se da a conocer por primera vez un tablero del corte estratigráfico del yacimiento paleolítico de San Isidro realizado por Emilio Rotondo que pertenece al material docente de la Escuela de Ingenieros de Caminos, Canales y Puertos de la Universidad Politécnica de Madrid, y que se halla en el Laboratorio de Geología. Es un tablero de las mismas características que los otros tres de este autor, localizados también en la capital. No conserva todos los elementos líticos, ni fósiles, pero su importancia radica en la documentación escrita asociada, donde Rotondo describe de manera detallada todos los niveles estratigráficos que se representan, contribuyendo a mejorar la información de este histórico yacimiento.

Se identifican 34 niveles entre los que se diferencian dos niveles arqueológicos: uno superior de arenas, arcillas (capa 26 con industria lítica, junto con capas 27-28-29-30 con restos de mamíferos fósiles) y uno inferior de gravas (capa 34 con industria lítica).

\section{AGRADECIMIENTOS}

Agradecemos de manera muy sincera las observaciones e indicaciones de los dos revisores anónimos que han servido para mejorar este trabajo.

\section{BIBLIOGRAFÍA}

Ariño, J. 2007: “El nacimiento del teléfono en España. Las dificultades del crecimiento de un nuevo sistema de comunicaciones, 1880 1924". Cuadernos de Historia Contemporánea 29: 35-56.

Aguirre, E. 1969: "Evolutionary history of the Elephant". Science 164: 1366-1376.
Aguirre, E. 2002: "Investigación y fondos de prehistoria madrileña en el Museo Nacional de Ciencias Naturales". En J. Panera y S. Rubio (eds.): Bifaces y Elefantes. La investigación del Paleolítico Inferior en Madrid. Zona Arqueológica 1, Museo Arqueológico Regional. Alcalá de Henares: 409-419.

Buffon, G. 1858: Historia Natural. Imprenta de Gaspar y Roig editores. Madrid.

Cacho, C. y Martos, J. A. 2002: "Colecciones paleolíticas de Madrid en el Museo Arqueológico Nacional". En J. Panera y S. Rubio (eds.): Bifaces y Elefantes. La investigación del Paleolítico Inferior en Madrid. Zona Arqueológica 1, Museo Arqueológico Regional. Alcalá de Henares: 183-407.

Gamazo, M. 2002: “Las colecciones paleolíticas del Manzanares y del Jarama del Museo de San Isidro". En J. Panera y S. Rubio (eds.): Bifaces y Elefantes. La investigación del Paleolítico Inferior en Madrid. Zona Arqueológica 1, Museo Arqueológico Regional. Alcalá de Henares: 359-381.

Gamazo Barrueco, M.; Oñate Baztán, P. y Hernández Pizarro, N. 2001: "Las terrazas de San Isidro. Nuevos datos sobre su extensión, características y cronología". Estudios de Prehistoria y Arqueología Madrileñas 11: 23-36.

Gómez Hernanz, J.; Márquez Mora, B.; Nicolás Checa, E.; Pérez-González, A. y Ruíz Zapata, B. 2005: "San Isidro (Madrid): 1862-2002. Nuevos hallazgos paleolíticos en la terraza de $+30 \mathrm{~m}$ del río Manzanares". Trabajos de Prehistoria 62 (1): 157-164; doi: 10.3989/tp.2005.v62.i1.

Graells, M. de la Paz 1897: Fauna Mastológica Ibérica. Memorias de la Real Academia de Ciencias Exactas, Físicas y Naturales de Madrid 17. Madrid: 1-806.

Hernández Pacheco, E. 1927: "Restos fósiles de grandes mamíferos en las terrazas del Manzanares y consideraciones respecto a éstas". Boletín de la Real Sociedad Española de Historia Natural 27: 449-455.

López Rodríguez, A. 2015: "Los hermanos Rotondo y Nicolau, coleccionistas arqueológicos de finales del siglo XIX y principios del XX". Espacio Tiempo y Forma. Serie Prehistoria y Arqueología 7: 211-238; doi: http://dx.doi.org/10.5944/etfi.7.2014.13997.

Mañas Martínez, J. 1983: Eduardo Saavedra, ingeniero $y$ humanista. Colegio de Ingenieros de Caminos, Canales y Puertos. Madrid.

Pérez de Barradas, J. 1929: "La colección prehistórica Rotondo". Actas y memorias de la Sociedad Española de Antropología, Etnografía y Prehistoria 8: 161-245.

Pérez de Barradas, J. 1941: "Nuevas investigaciones sobre el yacimiento de San Isidro (Madrid)". Archivo Español de Arqueología XIV: 277-303.

Prado, C. del 1864: Descripción Física y Geológica de la Provincia de Madrid. Junta Superior de Estadística. Madrid. 
Prado, C. del 1866: Discursos leídos ante la Real Academia de Ciencias Exactas, Físicas y Naturales en la recepción pública del Excmo. Sr. D. Casiano del Prado. Imp. de Eusebio Aguado. Madrid: 43-44.

Puche Riart, O. 1993: "La contribución de los ingenieros de minas a la Arqueología española: Los inicios de los estudios prehistóricos en España”. Boletín Geológico y Minero 104 (3): 94-128.

Rábano 2015, I.: Los cimientos de la Geología: la Comisión del Mapa Geológico de España (1849-1910). Instituto Geológico y Minero de España. Madrid.

Rudwick, M. J. S. 1976: "The emergence of a visual language for geological science, 1760-1840". History of Science 14: 149-195.

Sáenz Ridruejo, F. 1998: Los ingenieros de caminos. Cátedra Juan-Miguel Villar Mir, Colegio de Ingenieros de Caminos, Canales y Puertos, Colección ciencias, humanidades e ingeniería 47. Madrid.

Sáenz Ridruejo, F. 2005: Una historia de la Escuela de Caminos. 1802-1898. Fundación Agustín de Betancourt, Escuela Técnica Superior de Ingenieros de Caminos, Canales y Puertos. Madrid.

Salas Vázquez, E. 2008: Arqueología. América. Antropología. José Pérez de Barradas (1897-1981). Área de Gobierno de las Artes, Ayuntamiento de Madrid. Madrid.

Sánchez Miñana, J. y Villanueva García, B. 2014: “La primitiva oferta de equipo telefónico en España vista a través del concurso para la red del Ayuntamiento de Bilbao (1884)". Llull: Revista de la Sociedad Española de Historia de las Ciencias y de las Técnicas 2, 37, 79: 63-79.
Santonja, M. 1977: "Los bifaces del Cerro de San Isidro conservados en el Museo Arqueológico Nacional". Revista de Archivos, Bibliotecas y Museos LXXX: 147-182.

Santonja, M y Vega, G. 2002: “La investigación del Valle del Manzanares (1862-1975) en el contexto del Paleolítico español”. En J. Panera y S. Rubio (eds.): Bifaces y Elefantes. La investigación del Paleolítico Inferior en Madrid. Zona Arqueológica 1, Museo Arqueológico Regional. Alcalá de Henares: 243-275.

Sesé, C. y Soto, E. 2002a: "Vertebrados del Pleistoceno del Jarama y Manzanares". En J. Panera y S. Rubio (eds.): Bifaces y Elefantes. La investigación del Paleolítico Inferior en Madrid. Zona Arqueológica 1, Museo Arqueológico Regional. Alcalá de Henares: 318-337.

Sesé, C. y Soto, E. 2002b: “Catálogo de los yacimientos de vertebrados del Pleistoceno en las terrazas de los ríos Jarama y Manzanares". Bifaces y Elefantes. La investigación del Paleolitico Inferior en Madrid. Zona Arqueológica 1, Museo Arqueológico Regional. Alcalá de Henares: 431-457.

Vilanova i Piera, J. 1872a: Compendio de Geología. Imprenta de Alejandro Gómez Fuentenebro. Madrid.

Vilanova i Piera, J. 1872b: "Estudios sobre lo prehistórico español". Museo Español de Antigüedades. Impr. de T. Fortanet. Madrid: 187-230.

Wernert, P. y Pérez de Barradas, J. 1925: "El yacimiento paleolítico de San Isidro. Estudio bibliográficocrítico". Revista de la Biblioteca, Archivo y Museo del Ayuntamiento de Madrid II: 31-68 